\title{
Angiographic frame counts 90 minutes after streptokinase predict left ventricular function at 48 hours following myocardial infarction
}

\author{
J K French, I T Straznicky, B J Webber, P E Aylward, M J Frey, A A J Adgey,
} B F Williams, S C McLaughlin, H D White, for the HERO-1 Investigators

Department of Cardiology, Green Lane Hospital, Private Bag 92189, Auckland 1030, New Zealand

$\mathrm{J}$ K French

I T Straznicky

B J Webber

B F Williams

S C McLaughlin

H D White

Flinders

Cardiovascular

Centre, Adelaide,

South Australia

P E Aylward

Heart Center of

Sarasota, Florida, USA

M J Frey

Royal Victoria

Hospital, Belfast,

Northern Ireland, UK

A A J Adgey

Correspondence to:

Dr French.

email: johnf@ahsl.co.nz

A complete list of HERO-1 Investigators was published in Circulation

1997;96:2155-61.

Accepted for publication 31 July 1998

\begin{abstract}
Objective-To assess whether the 90 minute corrected thrombolysis in myocardial infarction frame count (CTFC) in the infarct related artery predicts left ventricular function at 48 hours in patients with myocardial infarction treated with aspirin, streptokinase, and either heparin or Hirulog.

Design and setting-Analysis of 251 patients with acute myocardial infarction enrolled in the international, multicentre Hirulog early reperfusion/occlusion (HERO-1) trial, who underwent both 90 minute coronary angiography and 48 hour left ventriculography.

Main outcome variables-The CTFC was determined in the infarct related artery $\mathbf{9 0}$ minutes after starting intravenous streptokinase $\left(1.5 \times 10^{6} \mathrm{U}\right.$ over 30 to 60 minutes), and compared with indices of left ventricular function assessed by contrast ventriculography at $\mathbf{4 8}$ hours.

Results-A CTFC of $\leqslant 27$ frames (previously reported mean $+2 \mathrm{SD}$ in coronary arteries of patients without acute infarction) occurred in $29 \%$ of infarct related arteries, and was associated with a lower infarct zone mean chord score $(-2.06 v$ $-2.54, p=0.01$ ), a lower fraction of chords $>2$ SD below normal (37\% v 51\%, $\mathbf{p}=\mathbf{0 . 0 0 5}$ ), and trends towards higher left ventricular ejection fractions $(60.9 \% v$ $58.2 \%, p=0.11$ ) and lower end systolic volumes $(50.1 \mathrm{ml} v 55.9 \mathrm{ml}, \mathrm{p}=\mathbf{0 . 2 3})$. A CTFC of $\leqslant 40$ at 90 minutes occurred in $50 \%$ of infarct related arteries, and was associated with a significantly lower mean chord score $(-2.20 v-2.60, \mathrm{p}=0.02)$, a smaller fraction of chords $>2$ SD below normal $(41 \% v 52 \%, p=0.025)$, a smaller end systolic volume $(49.1 \mathrm{ml} v 59.3 \mathrm{ml}$, $\mathbf{p}=0.02$ ), and a higher left ventricular ejection fraction $(60.4 \% \quad v \quad 56.5 \%$,

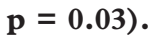

Conclusions-The 90 minute CTFC predicts left ventricular function at 48 hours following streptokinase. The CTFC associated with better ventricular function may be higher than values determined from a non-infarct population.

(Heart 1999;81:128-133)

Keywords: streptokinase; Hirulog; perfusion; ventricular function; frame count
Successful thrombolytic treatment for myocardial infarction is associated with rapid, complete, and sustained perfusion of the infarct related artery. ${ }^{12}$ The degree of perfusion achieved in the infarct related artery has traditionally been expressed as the thrombolysis in myocardial infarction (TIMI) trial flow grade, which correlates with survival after myocardial infarction. ${ }^{1-6}$ TIMI 3 flow (complete perfusion) 90 minutes after starting thrombolytic treatment is also associated with significantly better global and regional left ventricular function than TIMI 2 flow (partial perfusion) or TIMI 0-1 flow (occlusion) in the infarct related artery. ${ }^{3}$

While it provides prognostic information, questions have been raised about the reproducibility of TIMI flow grade assessment as there is significant interobserver variability of flow grading, particularly with respect to TIMI 2 flow. ${ }^{7}$ In order to quantify flow in a more objective manner, Gibson et al described the corrected TIMI frame count (CTFC). ${ }^{7}$ This method of angiographic assessment of perfusion counts the number of cineangiographic frames after initial contrast injection to achieve opacification of a defined distal landmark in the artery.

We examined whether infarct related artery flow at 90 minutes, as measured by the frame count, is associated with preservation of both global left ventricular function and regional wall motion in the infarct zone 48 hours after myocardial infarction.

\section{Methods}

The study group consisted of patients with acute myocardial infarction enrolled in the Hirulog early reperfusion/occlusion (HERO-1) trial, ${ }^{8}$ who had 90 minute coronary angiography and 48 hour coronary angiography and ventriculography, as specified by the trial protocol. Patients were randomised if they presented within 12 hours of symptom onset with chest pain of $>20$ minutes duration and had associated ST segment elevation on the electrocardiogram. All patients received aspirin (150-325 mg) and streptokinase $\left(1.5 \times 10^{6} \mathrm{U}\right.$ over 30 to 60 minutes), and were then randomised to receive either heparin $(5000 \mathrm{U}$ bolus followed by $1000-1200 \mathrm{U} / \mathrm{h}$ titrated to a therapeutic activated partial thromboplastin time), or Hirulog (either a $0.125 \mathrm{mg} / \mathrm{kg}$ bolus followed by $0.25 \mathrm{mg} / \mathrm{kg} / \mathrm{h}$ (low dose), or twice this dose (high dose)). ${ }^{8}$

Patients underwent coronary angiography at 90 minutes (interquartile range (IQR) 92 to 
Table 1 Baseline characteristics

\begin{tabular}{lll}
\hline Characteristic & HERO-1 & CTFC substudy \\
\hline Number & 412 & 251 \\
Age (years) (mean (SD)) & $61.4(11.9)$ & $60.8(11.4)$ \\
Female (\%) & 25 & 24 \\
Height (cm) (mean (SD)) & $134.1(50.6)$ & $140.7(48.1)$ \\
Weight (kg) (mean (SD)) & $82.0(26.4)$ & $81.7(25.9)$ \\
Heart rate (beats/min) (mean (SD)) & $75.2(17.1)$ & $74.8(16.0)$ \\
Diastolic blood pressure (mm Hg) (mean (SD)) & $132.0(21.3)$ & $133.5(21.0)$ \\
Previous infarction (\%) & 14 & 11 \\
Previous PTCA (\%) & 4 & 4 \\
Previous CABG (\%) & 3 & 2 \\
Hypertension (\%) & 30 & 27 \\
Smoking (\%) & 63 & 65 \\
Duration of streptokinase infusion (min) (mean (SD)) & $49.7(16.4)$ & $48.5(16.9)$ \\
Hirulog treatment (\%) & 66 & 69 \\
TIMI 3 flow at 90 minutes (\%) & 43.0 & 42.5 \\
Time to treatment (hours) (mean (SD)) & $3.94(2.7)$ & $4.01(2.7)$ \\
\hline
\end{tabular}

CABG, coronary artery bypass graft; CTFC, corrected TIMI frame count; PTCA, percutaneous transluminal coronary angioplasty; TIMI, thrombolysis in myocardial infarction trial.

110) after the start of streptokinase treatment, and this was repeated at 48 hours (IQR 43.4 to 51.2), along with contrast left ventriculography. The infarct related artery was identified on the basis of a combination of changes on the admission electrocardiogram, the location of wall motion abnormality on the ventriculogram, and the presence of thrombus in the artery. TIMI flow grading ${ }^{9}$ and frame count analyses were performed in a core angiographic laboratory by two experienced observers blinded to film sequence or clinical outcome. If flow grades differed between the two, a third independent observer adjudicated. Following the paper by Gibson et al, ${ }^{7}$ frame counts were corrected, including adjustment of cine acquisition rates to 30 frames/second. Frame counts in vein graft culprit vessels were corrected by a factor of 1.6 to account for their extra length. ${ }^{10}$

Contrast left ventriculography was performed in a $40^{\circ}$ right anterior oblique projection. Left ventricular end systolic volumes, end diastolic volumes, and ejection fractions were determined using a Cardiovascular Measurement System (Nuen, the Netherlands) quantitative angiography system. Regional left ventricular wall motion was assessed using the centreline method. ${ }^{11}$ Regional wall motion indices were expressed as either the mean chord motion score, or as the fraction of chords that were $>2$ SD below normal shortening within the infarct zone. Non-dominant circumflex infarct related arteries were excluded from this analysis, as the myocardial segments supplied are not profiled in the right anterior oblique projection.

\section{STATISTICAL ANALYSIS}

Results for continuous variables are expressed as mean (SD) or, if variables were not normally distributed, as median and interquartile range. Correlation coefficients were calculated for continuous variables, taking the natural logarithm of variables distributed in a nonparametric manner. Differences in mean and median values were compared using a paired $t$ test, unpaired $t$ test, and one way analysis of variance, as appropriate. Categorical variables are expressed in percentages, with comparisons made using a $\chi^{2}$ and Fisher's exact test. All $\mathrm{p}$ values are two tailed, with values $<0.05$ regarded as significant.
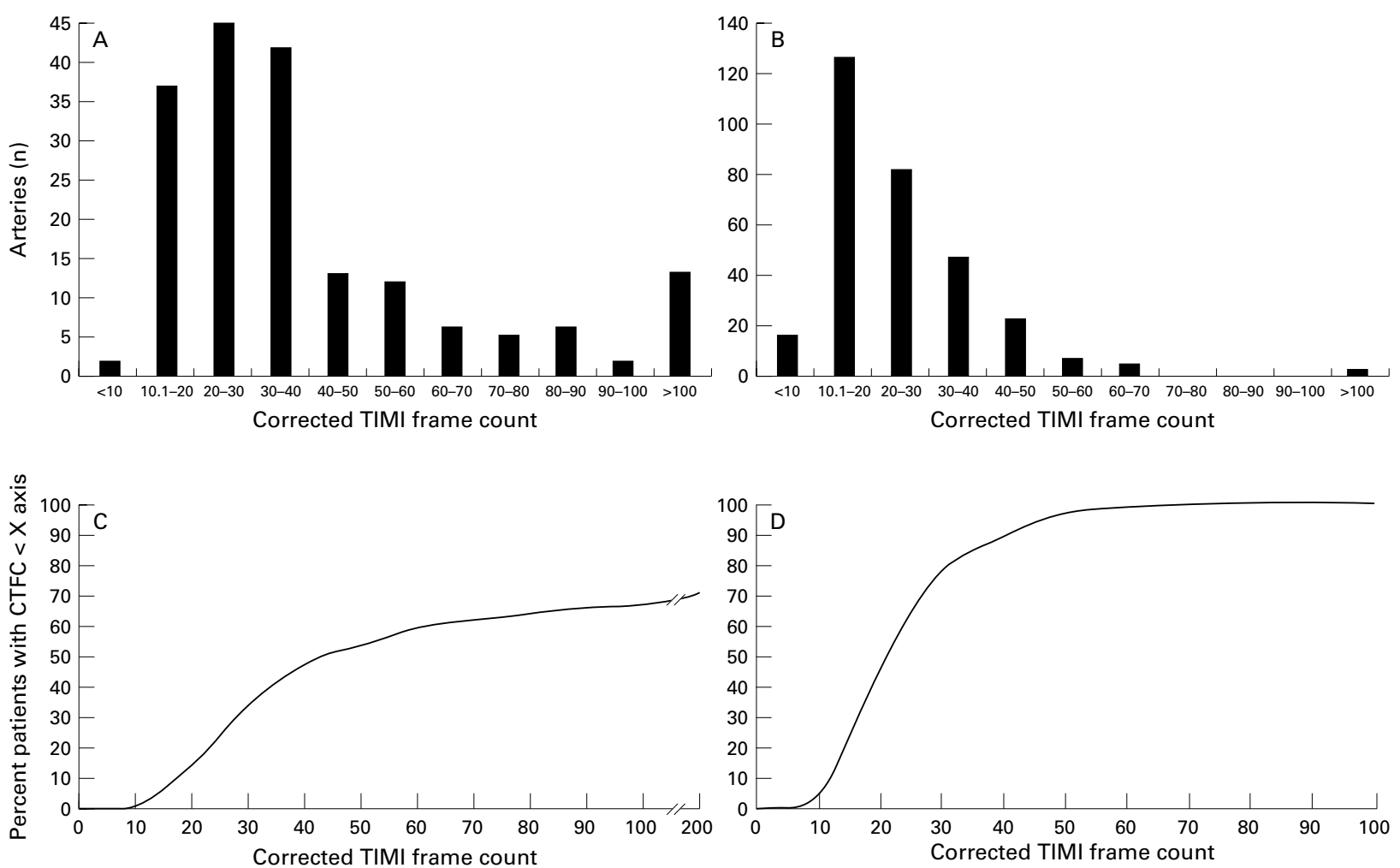

Figure 1 (A) Corrected TIMI frame count distribution among patent infarct related arteries at 90 minutes post-streptokinase. (B) Corrected TIMI frame count distribution among non-infarct related arteries at 90 minutes post-streptokinase. (C) Cumulative frequency distribution of corrected TIMI frame counts at 90 minutes in the infarct related artery. (D) Cumulative frequency distribution of corrected TIMI frame counts at 90 minutes in the non-infarct related artery. CTFC, corrected TIMI frame count; TIMI, thrombolysis in myocardial infarction trial. 
Table 2 Continuous variable analysis of corrected TIMI frame counts $v$ left ventricular function, imputing values for an occluded infarct related artery $(I R A)^{\star}$

\begin{tabular}{|c|c|c|c|c|}
\hline & $\begin{array}{l}\text { Patent IRA, } \\
C T F C \leqslant 100 \\
(n=159)\end{array}$ & $\begin{array}{l}\text { Patent }+ \text { occluded IRA, } \\
\max C T F C=284 \\
(n=251)\end{array}$ & $\begin{array}{l}\text { Patent }+ \text { occluded IRA, } \\
\max C T F C=200 \\
(n=251)\end{array}$ & $\begin{array}{l}\text { Patent }+ \text { occluded IRA, max } \\
\text { CTFC }=100(n=251)\end{array}$ \\
\hline \multirow[t]{2}{*}{ Ejection fraction } & $r=0.170$ & $r=0.0095$ & $r=0.098$ & $r=0.117$ \\
\hline & $p=0.03$ & $\mathrm{p}=0.217$ & $\mathrm{p}=0.203$ & $p=0.118$ \\
\hline \multirow[t]{2}{*}{ End systolic volume } & $r=0.235$ & $r=0.202$ & $r=0.202$ & $r=0.206$ \\
\hline & $\mathrm{p}=0.003$ & $\mathrm{p}=0.008$ & $\mathrm{p}=0.009$ & $\mathrm{p}=0.007$ \\
\hline \multirow[t]{2}{*}{ End diastolic volume } & $r=0.219$ & $r=0.256$ & $r=0.249$ & $r=0.239$ \\
\hline & $\mathrm{p}=0.007$ & $\mathrm{p}<0.001$ & $\mathrm{p}=0.001$ & $\mathrm{p}=0.002$ \\
\hline \multirow[t]{2}{*}{ Mean chord score } & $r=0.213$ & $r=0.142$ & $r=0.141$ & $r=0.164$ \\
\hline & $\mathrm{p}=0.02$ & $\mathrm{p}=0.065$ & $\mathrm{p}=0.065$ & $\mathrm{p}=0.032$ \\
\hline \multirow{2}{*}{ Chords $>$ 2SD below normal } & $r=0.190$ & $r=0.104$ & $r=0.110$ & $r=0.134$ \\
\hline & $\mathrm{p}=0.02$ & $\mathrm{p}=0.177$ & $\mathrm{p}=0.152$ & $\mathrm{p}=0.081$ \\
\hline
\end{tabular}

^Imputing values for occluded arteries involves assignment of the CTFC indicated in columns 2-4.

CTFC, corrected thrombolysis in myocardial infarction (TIMI) frame count.

\section{Results}

Of 412 patients randomised in the HERO-1 trial, ${ }^{8} 251$ had frame count analysis at $90 \mathrm{~min}$ utes and ventriculography at 48 hours. The 251 patients had similar baseline characteristics to the total HERO-1 population (table 1). Reasons for failure to proceed with ventriculography or frame count analysis included death, withdrawal of consent, performance of coronary angioplasty or bypass surgery after the first procedure, and technical unsuitability for frame counting. The infarct related artery was the left anterior descending coronary artery in $32 \%$, the right coronary artery in $57 \%$, and the circumflex artery in $10 \%$. Saphenous vein grafts were the infarct related artery in three cases.

CORRECTED TIMI FRAME COUNT AT 90 MINUTES AND 48 HOURS

The frame counts in infarct related and non-infarct related arteries are shown as distribution histograms (fig 1, A and B) and cumulative frequency curves (fig 1, C and D). The median frame count in patent infarct related arteries was 31.2 frames (IQR 22.7 to 51.0 ) at 90 minutes and 23.3 frames (IQR 17.3 to 33.2 ) at 48 hours ( $\mathrm{p}<0.001$; mean (SD) 42.5 (33.7) and 29.1 (21.9), respectively). Among noninfarct related arteries, the median frame count at 90 minutes was 20.9 frames (IQR 15.1 to 28.8; mean (SD) 24.0 (13.5)) compared with 22.8 frames (IQR 16.0 to 32.4 ; mean (SD) 25.9 (13.6); $\mathrm{p}=0.03$ ) at 48 hours.
FRAME COUNT AND LEFT VENTRICULAR

FUNCTION AS CONTINUOUS VARIABLES

Because of the skewed distribution of frame counts in patent infarct related arteries (fig 1A), the natural logarithm of the frame count was used for continuous variable analysis. As occluded arteries have no numerical frame count value, only patent arteries $(n=173)$ were included in the initial analysis. A correlation was seen between frame count and end systolic volume $(r=0.202, \mathrm{p}=0.008)$ and end diastolic volume $(r=0.256, \mathrm{p}<0.001)$. There was no correlation between frame count and mean chord score in the infarct zone $(r=0.142, \mathrm{p}=0.07)$, the fraction of chords with motion $>2$ SD below normal $(r=0.104$, $\mathrm{p}=0.18)$ or ejection fraction $(r=0.095$, $\mathrm{p}=0.22)$.

It is uncertain how best to include data from occluded arteries in a continuous variable analysis. Excluding occluded arteries excludes ventricular function data from those patients who may have the greatest degree of ventricular dysfunction. We therefore performed a conservative analysis, assigning occluded infarct related arteries arbitrary values, including the maximum frame count of patent infarct related arteries (284 frames), 200 frames, and 100 frames. The correlations between frame count and indices of ventricular function were lost, however, owing to clustering at the maximum value (table 2). Logarithmic and inverse transformations did not alter these correlations. The strongest correlations between frame count

Table 390 minute frame count v left ventricular function at 48 hours

\begin{tabular}{|c|c|c|c|c|}
\hline & $\begin{array}{l}C T F C \leqslant 27 \\
(n=70)^{*}\end{array}$ & $\begin{array}{l}C T F C>27 \\
(n=163)\end{array}$ & $95 \% C I$ & $p$ Value \\
\hline Mean chord score & $-2.06(1.22)$ & $-2.54(1.32)$ & 0.12 to 0.84 & 0.01 \\
\hline Chords > 2 SD below normal (\%) & $37.0(34.0)$ & $51.0(35.0)$ & -24 to -4 & 0.005 \\
\hline End diastolic volume $(\mathrm{ml})$ & $122.7(49.2)$ & $131.1(59.6)$ & -23.1 to 6.3 & 0.26 \\
\hline End systolic volume (ml) & $50.1(33.2)$ & $55.9(36.0)$ & -15.4 to 3.8 & 0.23 \\
\hline \multirow[t]{2}{*}{ Ejection fraction (\%) } & $60.9(11.9)$ & $58.2(12.5)$ & -0.7 to 6.1 & 0.11 \\
\hline & $\begin{array}{l}C T F C \leqslant 40 \\
(n=119)\end{array}$ & $\begin{array}{l}C T F C>40 \\
(n=114)\end{array}$ & $95 \% C I$ & $p$ Value \\
\hline Mean chord score & $-2.20(1.27)$ & $-2.60(1.29)$ & 0.06 to 0.74 & 0.022 \\
\hline Chords > 2 SD below normal (\%) & $42.0(35.0)$ & $52.0(34.0)$ & -19 to -1 & 0.025 \\
\hline End diastolic volume (ml) & $120.3(51.2)$ & $137.0(60.9)$ & -30.9 to -2.3 & 0.024 \\
\hline End systolic volume (ml) & $49.1(33.2)$ & $59.3(36.6)$ & -19.2 to -1.3 & 0.024 \\
\hline Ejection fraction (\%) & $60.7(11.7)$ & $57.3(12.8)$ & 0.3 to 6.6 & 0.03 \\
\hline
\end{tabular}

*Patients with incomplete data were not included, for example those with non-dominant circumflex arteries.

Values are mean (SD). CI, confidence interval; CTFC, corrected thrombolysis in myocardial infarction trial (TIMI) frame count. 

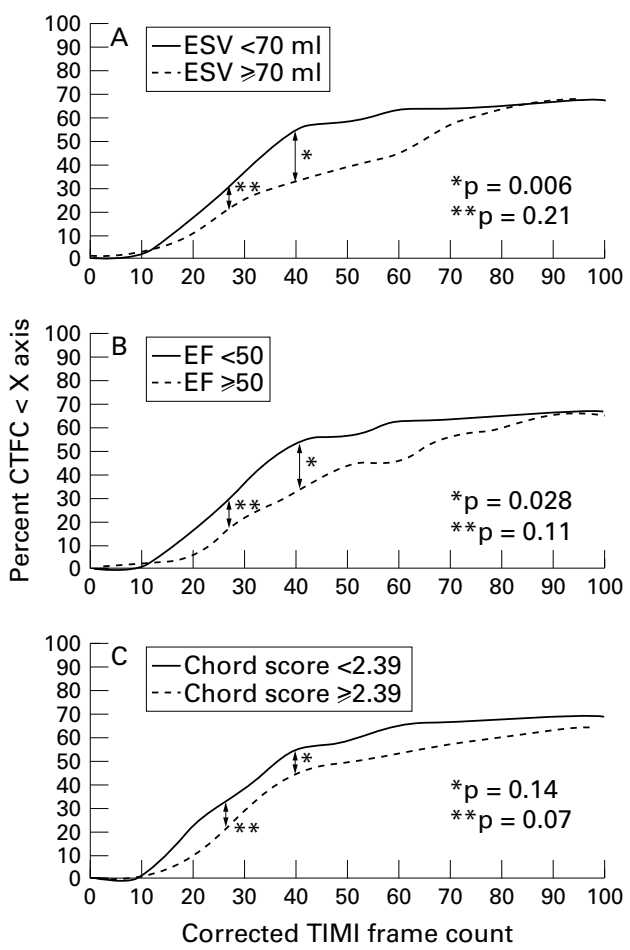

Figure 2 (A) Cumulative frequency distribution of corrected TIMI frame count at 90 minutes in patients with end systolic volumes of $\geqslant 70 \mathrm{ml} v<70 \mathrm{ml}$ at 48 hours. (B) Cumulative frequency distribution of corrected TIMI frame count at 90 minutes in patients with ejection fraction of $\geqslant 50 \%$ v $<50 \%$ at 48 hours. (C) Cumulative frequency distribution of corrected TIMI frame count at 90 minutes in patients with a chord motion score of $>2 S D v$ $<2 S D$ from mean in the infarct zone at 48 hours. CTFC, corrected TIMI frame count; EF, ejection function; ESV, end systolic volume; TIMI, thrombolysis in myocardial infarction trial.

and indices of ventricular function were seen when patients with patent infarct related arteries and a frame count of $\leqslant 100$ were subjected to continuous variable analysis (table 2).

FRAME COUNT AND LEFT VENTRICULAR FUNCTION AT DISCRETE CUT OFF POINTS Ventricular function data were also analysed using prespecified frame count cut off points. The cut off points chosen were $\leqslant 27$ frames, which is the reported $95 \%$ confidence limit of the mean frame count in a non-infarction population, ${ }^{7}$ and $\leqslant 40$ frames, which is the level associated with increased late reocclusion postinfarction. ${ }^{12}$ A frame count of $\leqslant 27$ at 90 minutes was seen in $29 \%$ of all infarct related arteries, compared with $43 \%$ assessed as having TIMI 3 flow. A frame count of $\leqslant 40$ was seen in $50 \%$ of infarct related arteries.

On left ventriculography at 48 hours, patients with a frame count of $\leqslant 27$ at 90 minutes had a significantly lower mean chord score and fraction of chords with motion $>2 \mathrm{SD}$ below normal in the area at risk on the 48 hour ventriculogram, compared with patients with a frame count $>27$ (table 3 ). There was a trend towards a higher left ventricular ejection fraction and lower end diastolic and end systolic volumes in these patients. No patient with a frame count of $\leqslant 27$ suffered reocclusion of the infarct related artery in the first 48 hours, while reocclusion occurred in $6 \%$ of patients with a frame count of $>27$ (NS). There was no difference in either global or regional left ventricular function between patients with a frame count of $\leqslant$ or $>27$ at 48 hours.

Both regional and global left ventricular functional indices were significantly better in those achieving a frame count of $\leqslant 40$ at 90 minutes compared with those with a higher frame count (table 3). Cumulative frequency curves were constructed, comparing the frequency of frame counts at 90 minutes in patients with an ejection fraction of $\geqslant 50 \% v$ $<50 \%$, end systolic volume of $\geqslant 70 \mathrm{ml} v<70$ $\mathrm{ml}$, and mean chord motion of $>2 \mathrm{SD} v<2$ $\mathrm{SD}$ below normal at 48 hours (fig $2, \mathrm{~A}-\mathrm{C}$ ). More patients with an ejection fraction of $\geqslant 50 \%$ and an end systolic volume of $<70 \mathrm{ml}$ at 48 hours had a frame count of $\leqslant 40$ at 90 minutes ( $p=0.028$ and $p=0.006$, respectively), compared with patients with either a lower ejection fraction or a higher end systolic volume. Patients with TIMI 3 flow at $90 \mathrm{~min}-$ utes had better global and regional left ventricular function than patients with TIMI 0-2 flow (table 4).

\section{Discussion}

The 90 minute TIMI flow grade has become the gold standard for angiographic assessment of reperfusion following thrombolytic treatment for myocardial infarction. In a metaanalysis of five angiographic studies comparing infarct related artery patency with outcome, Anderson et al reported that patients with TIMI 3 flow at 90 minutes had significantly better short term survival, with a 30 day mortality odds ratio of 0.45 (95\% confidence interval 0.34 to $0.61, \mathrm{p}<0.001)^{3}$ compared with those with less than TIMI 3 flow. The ejection fraction was $4 \%$ higher in patients achieving TIMI 3 flow at 90 minutes compared with patients with TIMI $0-2$ flow. ${ }^{3}$ In the global use of strategies to open occluded coronary arteries (GUSTO-1) trial, regional wall motion was significantly better in patients with TIMI 3 $v$ TIMI 2 flow at 90 minutes. ${ }^{1}$ No differences in either survival or left ventricular function have been shown between lower grades of TIMI flow. ${ }^{3}$

Table 490 minute infarct artery flow v left ventricular function at 48 hours

\begin{tabular}{llllll}
\hline Variable & $\begin{array}{l}\text { TIMI 0-1 } \\
(n=67)\end{array}$ & $\begin{array}{l}\text { TIMI } \\
(n=73)\end{array}$ & $\begin{array}{l}\text { TIMI 0-2 } \\
(n=140)\end{array}$ & $\begin{array}{l}\text { TIMI 3 } \\
(n=103)\end{array}$ & $\begin{array}{l}p \text { Value } \\
(\text { TIMI 3 v 0-2) }\end{array}$ \\
\hline Mean chord score & $-2.58(1.09)$ & $-2.48(1.56)$ & $-2.53(1.56)$ & $-2.26(1.31)$ & 0.03 \\
Chords > 2 SD below normal (\%) & $53.2(33.1)$ & $49.0(37.1)$ & $50.7(35.2)$ & $39.6(35.6)$ & 0.02 \\
End diastolic volume (ml) & $123.7(47.0)$ & $140.6(71.6)$ & $132.0(61.3)$ & $121.0(41.8)$ & 0.09 \\
End systolic volume (ml) & $51.3(26.2)$ & $63.4(44.9)$ & $57.3(37.5)$ & $48.0(25.8)$ & 0.02 \\
Ejection fraction (\%) & $58.8(11.9)$ & $56.5(14.1)$ & $57.7(13.1)$ & $61.1(11.4)$ & 0.01 \\
\hline
\end{tabular}

*Patients with incomplete data were not included.

Values are mean (SD). TIMI, thrombolysis in myocardial infarction trial. 
In analysis of clinical trial cineangiograms, significant differences have been found in TIMI flow grade assessment between local investigators and core angiographic laboratories. In an analysis of core versus local angiographic assessment of 90 minute TIMI flow grades in the TIMI 4 trial, there was excellent agreement in the assessment of TIMI grades 0 or 1 , moderate agreement with respect to TIMI 3 flow, but poor agreement in the assessment of TIMI 2 flow (only $52 \%$ ). ${ }^{7}$ This is not surprising as the assessment of TIMI flow is subjective and is affected by the length of the artery being assessed. ${ }^{13} 14$

The corrected TIMI frame count is an objective and reproducible measure of flow in the infarct related artery, is quantifiable, and relies less on observer experience. Thus it has the potential to become the new gold standard for the assessment of coronary reperfusion. ${ }^{13}{ }^{14}$ Before this can occur, however, the 90 minute frame count requires further validation with respect to clinical and angiographic outcomes following infarction.

In this study we have shown that a frame count of $\leqslant 27$ at 90 minutes after thrombolytic treatment is associated with less regional left ventricular dysfunction and a trend towards lower end systolic volume and improved ejection fraction at 48 hours. We have also shown that up to a frame count of 100 , there is a significant correlation between the frame count at 90 minutes and all measured indices of ventricular function at 48 hours. No patient with a frame count of $\leqslant 27$ at 90 minutes had reocclusion in the first 48 hours.

Up to a frame count of 100 , we noted a correlation between increasing frame count and a worsening of indices of global and regional ventricular function. The relation between frame count and ventricular function appeared to weaken above 100 frames. The reason for this is not clear. It may be that functionally, a frame count above 100 is equivalent to an occluded artery (TIMI $0-1$ flow), and thus further prolongation above this value is not associated with greater ventricular dysfunction. It may, however, simply reflect the relatively small number of infarct related arteries with a frame count above this value.

We attempted to correct for exclusion of occluded infarct related arteries by imputing arbitrary values for these vessels. Values imputed included 100 frames, a value that requires approximately $5 \%$ of patent infarct related arteries to be adjusted downwards, 200 frames, which requires only $0.8 \%$ of patent infarct related arteries to be adjusted downwards, and 284 frames, the maximum value for patent infarct related arteries in this study. The correlation between ventricular function and frame count weakened with all of these assumptions.

The threshold value of frame count necessary for reperfusion to maintain myocyte function remains to be defined. Twenty seven frames is the mean +2 SD normal coronary artery flow, based on an analysis of coronary flow in 78 patients who did not have myocardial infarction. ${ }^{7}$ The use of this cut off point has several limitations. First, in our study a higher frame count cut off point of 40 frames more strongly predicted 48 hour left ventricular function. Second, we have previously reported that a frame count of $>40$, rather than $>27$, at four weeks is associated with increased late reocclusion. ${ }^{12}$ Finally, a frame count which is normal in a population of patients without acute infarction may not be appropriate to determine the efficacy of reperfusion during acute infarction. The frame count is significantly prolonged during acute infarction, even in the non-infarct related artery, suggesting that infarction affects flow in the entire coronary vasculature. ${ }^{7}$ The use of a mean rather than the median to define the limits of statistical normality may not be appropriate for a variable such as frame count, which has a skewed rather then a normal distribution (fig 1, A and B). In practice, whatever constitutes a statistically "normal" frame count may not necessarily be the frame count which best predicts favourable or poor outcome, and a single numerical cut off may be an oversimplification, as the optimal value may vary according to a number of factors, including age, sex, coronary anatomy, collaterals, and the duration of occlusion before reperfusion.

In this study, the frame count in the non-infarct related artery failed to improve with time, and in fact slowed between 90 minutes and 48 hours post-thrombolysis, which contrasts with the findings of Gibson et al. ${ }^{7}$ There are several possible explanations for this. The timing of the second angiogram differed between the two studies, with angiography being performed at 48 hours in our study rather than at 36 hours. Different thrombolytic and antithrombotic regimens were used in the two studies, a larger number of patients was assessed in our study, and the baseline characteristics of the patient groups and the distribution of non-infarct related arteries may also have been different. The slowing of non-infarct related artery flow may be related to the effect of heightened sympathetic tone on coronary resistance vessels. ${ }^{12}$ This heightened state of resistance is thought to persist for up to a week. $^{15}$

\section{STUDY LIMITATIONS}

Only patients with angiograms at both 90 minutes and 48 hours were included in our sample, and there may have been a selection bias as a result. Those excluded may have been the more seriously ill patients, who may have died or required urgent revascularisation before a second study was possible. Such patients are likely to have had poor reperfusion in the infarct related artery, and their exclusion may have led to an underestimation of the association between frame count and left ventricular function. Additionally, ventriculography at 48 hours was specified by the HERO-1 trial protocol, and abnormalities of ventricular function at this time may reflect ventricular stunning rather than myocyte necrosis, ${ }^{16}$ and therefore may not give a true indication of long term ventricular function. 
CONCLUSIONS

A lower 90 minute frame count is associated with improved indices of left ventricular function at 48 hours in patients with myocardial infarction treated with streptokinase. The optimum frame count cut off point which best predicts patients who will have preserved ventricular function may be higher than a normal value $(\leqslant 27)$ determined from a non-infarct population of patients.

Frame counting may in the future become the gold standard for accurately and reproducibly determining the success of reperfusion with thrombolytic treatment after myocardial infarction. Full validation of the use of the corrected TIMI frame count requires studies with more patients to show a relation between a lower frame count and increased survival, an outcome for which our study was not powered.

Supported in part by a grant from the Health Research Council of New Zealand.

1 The GUSTO Angiographic Investigators. The effects of tissue plasminogen activator, streptokinase, or both on coronary artery patency, ventricular function, and survival after acute myocardial infarction. N Engl f Med 1993;329:161522.

2 Simes RJ, Topol EJ, Holmes DR, et al, for the GUSTOInvestigators. Link between the angiographic substudy and
mortality outcomes in a large randomized trial of myocarmortality outcomes in a large randomized trial of myocardial reperfusion: importance of early and complet
artery reperfusion. Circulation 1995;91:1923-8.

3 Anderson JL, Karagounis LA, Califf RM. Metaanalysis of five reported studies on the relation of early coronary patency grades with mortality and outcomes after acute myocardial infarction. Am $\mathcal{F}$ Cardiol 1996;78:1-8.

4 Vogt A, Von Essen R, Tebbe U, et al. Impact of early perfusion status of the infarct related artery on short-term mortality after thrombolysis for acute myocardial infarction: retro thective analysis of four German multiceninfarction: retrospective analysis of four Germa
tre studies. F Am Coll Cardiol 1993;21:1391-5.

5 Anderson JL, Karagounis LA, Becker LC, et al, for the TEAM-3 Investigators. TIMI perfusion grade 3 but not grade 2 results in improved outcome after thrombolysis for myocardial infarction. Circulation 1993;87:1829-39.

6 Ross AM, Coyne KS, Moreyra E, et al, for the GUSTO-I Angiographic Investigators. Extended mortality benefit of early postinfarction reperfusion. Circulation 1998;97:154956.

7 Gibson CM, Cannon CP, Daley WL, et al, for the TIMI 4 Study Group. TIMI frame count: a quantitative method of assessing coronary artery flow. Circulation 1996;93:879-88.

8 White HD, Aylward PE, Frey MJ, et al, on behalf of the HERO Trial Investigators. A randomized, double-blind comparison of Hirulog versus heparin in patients receiving streptokinase and aspirin for acute myocardial infarction (HERO). Circulation 1997;96:2155-61.

9 Chesebro JH, Knatterud G, Roberts R, et al. Thrombolysis in myocardial infarction (TIMI) trial, phase I: a comparison between intravenous tissue plasminogen activator and intravenous streptokinase: clinical findings through hospital discharge. Circulation 1987;76:142-54.

10 Al-Mousa E, Dodge JT, Rizzo M, et al. The TIMI frame count in saphenous vein grafts. Am Heart 7 1998:135: $323-8$

11 Cross DB, Ashton NG, Norris RM, et al. Comparison of the effects of streptokinase and tissue plasminogen activator on regional wall motion after first myocardial infarction: analysis by the centerline method with correction for area at risk. F Am Coll Cardiol 1991;17:1039-46.

12 French JK, Ellis CJ, Webber BJ, et al. Abnormal coronary flow in infarct arteries 1 year after myocardial infarction is predicted at 4 weeks by corrected thrombolysis in myocardial infarction (TIMI) frame count and stenosis severity. Am f Cardiol 1998;81:665-71.

13 White CW. Simplicity's virtues scorned. Precision comes to TIMI flow grading and the results are.....surprising [editorial]. Circulation 1996;93:853-6.

14 French JK, Ellis CJ, White HD. Corrected TIMI frame count. The new gold standard? Aust NZ f Med. count. The new

15 Uren NG, Crake T, Lefroy DC, et al. Reduced coronary vasodilator function in infarcted and normal myocardium after myocardial infarction. N Engl f Med 1994;331:222-7.

16 Braunwald E, Kloner RA. The stunned myocardium: prolonged post-ischemic ventricular dysfunction. Circulation 1982;66:1146-9. 